\title{
A comprehensive review on phytochemical, pharmacological and therapeutic properties of Agrimonia eupatoria L.
}

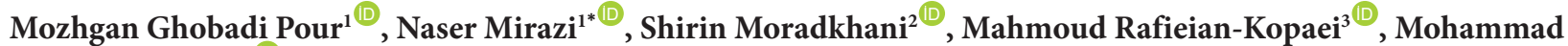 \\ Rahimi-Madiseh $^{3}$ \\ ${ }^{1}$ Department of Biology, Faculty of Basic Sciences, Bu- Ali Sina University, Hamedan, Iran \\ ${ }^{2}$ Department of Pharmacognosy, Faculty of Pharmacy, Hamadan University of Medical Sciences, Hamadan, Iran \\ ${ }^{3}$ Medical Plants Research Center, Basic Health Sciences Institute, Shahrekord University of Medical Sciences, Shahrekord, Iran
}

\section{A R T I C L E I N F O}

Article Type:

Review

\section{Article History:}

Received: 3 August 2020

Accepted: 10 October 2020

Keywords:

Agrimonia eupatoria

Agrimony

Flavonoid

Medicinal plant

Oxidative stress

Rosaceae

\begin{abstract}
A B S T R A C T
Agrimonia eupatoria is a perennial herb belonging to the Rosaceae family that all its parts are used to treat various diseases. In this article, we aimed to present a comprehensive review on the phytochemical, pharmacological, and therapeutic effects of this plant. We searched various databases and summarized the data documented in literature from 1976 to 2020. Agrimonia eupatoria has effects on various kinds of cancer, oxidative stress, diabetes mellitus, hepatitis B, and liver damage. It also has anti-adhesive, antibacterial, antimicrobial, and wound healing properties. It induces nitric oxide and inhibits pro-inflammatory cytokines production. Phytochemical studies related to this plant has led to isolation and identification of tannins, coumarins, and flavonoids as the most active chemicals with biological effects. Based on this comprehensive review about Agrimonia eupatoria, there will be more opportunities for investigators to search and discover ways to use bioactive agents of this herb to develop new Agrimony based medicines.
\end{abstract}

Implication for health policy/practice/research/medical education:

This review offers a comprehensive insight into the phytochemical, pharmacological, therapeutic activities, and safety of Agrimonia eupatoria L., and demonstrates that it can be used as a reliable source for preparation of new drugs..

Please cite this paper as: Ghobadi Pour M, Mirazi N, Moradkhani Sh, Rafieian Kopaei M, Rahimi-Madiseh M. A comprehensive review on phytochemical, pharmacological and therapeutic properties of Agrimonia eupatoria L. J Herbmed Pharmacol. 2021;10(1):14-30. doi: 10.34172/jhp.2021.02.

\section{Introduction}

Compared to conventional drugs, medicinal plants usually have low toxicity. Furthermore, in spite of the availability of all kinds of medicines and all health facilities, due to the high costs of hospital care or because of cultural beliefs and historical customs in most of the undeveloped countries, people still trust herbal medicines and some of the herbs still have kept their popularities (1).

Agrimonia eupatoria from the Rosaceae family, known as "Common Agrimony", is an erect perennial plant (30$60 \mathrm{~cm}$ in height). It is native to mainland Europe and found across Asia Minor and North Africa (2).

It is traditionally used as an antiadhesive, antibacterial $(3,4)$, antioxidant, astringent $(5,6)$, anti-inflammatory (7-9), and hepatoprotective (10) agent. It is also used for bed-wetting (11), treatment of hemorrhagic colitis, liver and urinary diseases $(6,12)$, cancer (13), acute diarrhea, diabetes mellitus, inflammation of oral and pharyngeal mucosa $(14,15)$, and the hepatitis B virus (16). In this article we aimed to present a comprehensive review on phytochemical, pharmacological, and therapeutic properties of this plant.

\section{Botany}

Scientific Classification

The scientific classification of A. eupatoria (Figure 1) is as follows:

Super kingdom: Eukaryota

Kingdom: Viridiplantae

Phylum: Streptophyta

Subphylum: Streptophytina

Class: Magnoliopsida 


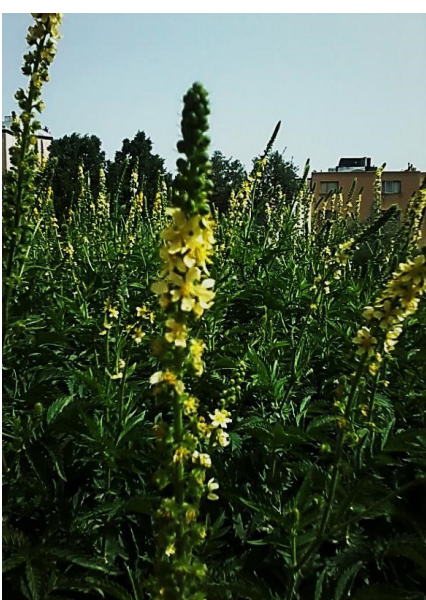

Figure 1. Agrimonia eupatoria.

Order: Rosales

Family: Rosaceae

Subfamily: Rosoideae

Tribe: Sanguisorbeae

Subtribe: Agrimoniinae

Genus: Agrimonia

Species: Agrimonia eupatoria

Names of Aigremoine eupatoria in different languages

Names of A. eupatoria in different languages are as follows: Anglo-Saxon: Garclive.

Arabic: Eufātorion, Shajarat ol barāghith, Shawkat Montenah, A'rang, Aghremun, Ghāfath, Hashisht ol ghāfath.

Chinese: Da Hua Long Ya Cao.

English: Common Agrimony, Cocklebur, Stickle wort, Liver Wort, Church, Steeples, Beggar's Ticks, White Tansy, Wild Tansy.

Farsi: Moshkaniyeh, Dava-ye- Jegar, Gole Khole, Gol Roghane Kaah, Gole, Chasbak, Alafe Geloo Dard.

French: Herbe d'eupatoire, Aigremoine commune, Eupatoire des anciens, Thé des bois, Souberiette, Aigremoine eupatoire, Herb de Saint Guillaume, Herb de la mère.

Germany: Kleiner odermenning, Leberkraut, Agremomen. Greek: Eupatorios.

Italian: Erba vettonica, Santonia, Erba da andata, Agrimonia Eupatoria.

Russian: Repejniček Aptechnyı̆ (17).

Solvak: Repík lekársky, Repík, repíček, starček, konopínec, útrobník, boží bič (18).

Spanish: Agrimonia, Hierba bacera, Hierba del podabor. Swedish: Småborre.

Turkish: Egir otu, Kasik otu, Kizil yaprak, Koyun otu (17).

In Iranian traditional medicine (ITM), there are two plants that are called Ghafath; the amateur users usually do not notice this. Users should look for a description of the plant in each book before using it and should notice which one the writer means. The agrimonium sp. (Rosaceae) Ghafath has yellow flowers, but the second one has pink or reddish flowers with the scientific name of Eupatorium cannabinum L. (Asteraceae) (19). The second Ghafath named Ghafath e kanafi which means "hemp agrimony" in Persian (20), "hemp agrimony" is Eupatorium cannabinum common name in English.

\section{Description}

Agrimonia eupatoria is a plant with bear pinnate, toothed alternate, leaves with undersides like velvet and small pairs between larger pairs with erect, reddish and pubescent stems (50-150 cm high). It has basal leaves as a rosette, and on its long, slender spikes grow five small petals of bright yellow flowers. The fruits are small, cone-shaped, enclosed in a calyx-tube with bristle. The bristles with hook enable the dispersal of the seeds on animal fur. It also spreads vegetatively by stout, woody, deep-lying rhizomes (21).

Some insects associate only with this herb like an eriophyoid mite, Aculus castriferrei n. sp (22) and Stigmella aeneofasciella Larvae (23). This is why Agrimony is called "Shajarat ol barāghith" in Arabic, which means the tree of fleas in English.

\section{Related species}

Agrimonia pilosa and A. viscidula Bunge are used in China for comparable conditions (24). The German Commission E Pharmacopoeial Monograph enables the use of $A$. procera as a second precious source of Agrimoniae herba. Granica et al have shown, based on their collected data, that a legitimate source of plant materials for drug preparation is A. procera (Fragrant agrimony) (25).

\section{Cultivation and habitat}

Agrimony is a native European herb mostly found in marshes, on wasteland, and wet meadows (1). It is one of the most common species of dry grasslands in the ex-arable land of the SE Czech Republic (26) and Slovak Republic (18). Thus, it has been found in the Hindu Kush Mountains of Pakistan (27), as well as Western Himalaya, and India (28). It is harvested during its flowering stage in summer (1).

Agrimonia eupatoria does not reach the edge of its distribution. It displayed dynamic changes in leaf morphology in response to tree shade and in elongation of stems and inflorescences because of herbaceous shade. Because of $A$. eupatoria's high active plasticity, it can maintain constant shoot growth in a variety of light conditions (29). The normal distance in cultivation on seed yield is $4 / \mathrm{m}-2$ (30). A. eupatoria best germination results obtained at $20^{\circ} \mathrm{C}$ at 12 -hour daily photoperiod, 5.5 $\mathrm{cm}$ root cuttings develop stems and/or roots at $15^{\circ} \mathrm{C}$ and $25^{\circ} \mathrm{C}(31)$. 
Table 1. Macroscopic characters of $A$. eupatoria

\begin{tabular}{|c|c|c|}
\hline A. eupatoria & & Characters \\
\hline \multirow{3}{*}{ Inflorescence } & & Elongate raceme \\
\hline & & Terminal \\
\hline & & September-October \\
\hline \multirow{3}{*}{$\begin{array}{l}\text { Stem } \\
\text { structures }\end{array}$} & Length & $30-60 \mathrm{~cm}$ high \\
\hline & \multirow{2}{*}{ Surface } & Villous \\
\hline & & Reddish \\
\hline \multirow{10}{*}{ Leaves } & & Alternate \\
\hline & & Pinnatifid \\
\hline & \multirow{3}{*}{ Segments } & Oval-lanceolate \\
\hline & & Deeply dentate \\
\hline & & Irregular \\
\hline & \multirow{2}{*}{$\begin{array}{l}\text { Underside } \\
\text { villous }\end{array}$} & White \\
\hline & & Without glands \\
\hline & \multirow{3}{*}{ Stipules } & Purplish \\
\hline & & Clasping \\
\hline & & Incised dentate \\
\hline \multirow{13}{*}{ Flowers } & \multirow{4}{*}{ Receptacle } & Small \\
\hline & & Yellow \\
\hline & & Concave \\
\hline & & A narrow opening \\
\hline & \multirow{2}{*}{ Calyx tube } & Furrowed \\
\hline & & Curved spurs at the tip \\
\hline & Lobes & 5 \\
\hline & \multirow{3}{*}{ Petals } & 5 \\
\hline & & Small \\
\hline & & Imbricate \\
\hline & \multirow{2}{*}{ Stamens } & $5-15$ \\
\hline & & Slender \\
\hline & Carpel & A single at maturity \\
\hline \multirow[t]{2}{*}{ Fruit } & \multirow[t]{2}{*}{ An achene } & $\begin{array}{l}\text { Surrounded with the mithered } \\
\text { receptacle }\end{array}$ \\
\hline & & Crowned with the persistent calyx \\
\hline \multirow{2}{*}{ Taste } & Bitter & \\
\hline & Astringent & \\
\hline
\end{tabular}

Macroscopic Types

Agrimonia eupatoria's characters are given in Table 1 (24).

Microscopic characters

Agrimonia eupatoria's characters are given in Table 2 (32).

\section{Ethnopharmacology}

The plant has a "cool" and "drying" characteristics. All parts, mostly the aerial parts of the plant are used for various diseases (33).

History and folklore

Agrimonia eupatoria was named by Mithridates Eupator, a king of Pontus, a famous plant collector and botanical text author in Greek. Thus, Pliny records, 'it has gotten credit and reputation by a king, as may appear by the name'. Many centuries later, Fuchs named it 'Hepatorium', because of its protective effects on the liver. Until the $18^{\text {th }}$ century Agrimony was known in the Linnaean classification under the title 'Eupatorion', so Dioscorides (IV 41) named it the same (21).

Traditional uses

Agrimonia eupatoria was worshiped in the Kysuce region (18). Since then, people have found various methods for using Agrimony for almost all parts of the body illnesses as shown in Table 3. A. eupatoria has been used since Saxon times. It was the primary ingredient of a battlefield cure for bullet wounds called "Arquebusade water" in the $15^{\text {th }}$ century. Agrimony healing power is now credited with the herb's high silica content (33). A. eupatoria, known as Ghafath in Iranian traditional medicine (ITM), has been repeatedly used for liver strengthen and it was well documented as an outstanding liver tonic. It is very useful and extensively recommended in Iranian medical literature (34), and used mainly to treat liver disorders (35). It is one of the most important ingredients of ITM tablets like "Qurse Rewand" which means "Rhubarb tablet" in Iranian dialect. Its effects on rats have been recently documented as significant hepatocurative agent and showed signs of recovery and regeneration in damaged liver cells (36).

It is used as antibacterial, astringent, aggregant, antipyretic, diuretic, antidiabetic, anti-inflammatory, antiseptic, antiviral, candidicide, vermifuge, tonic, uricolytic, depurative, cholagogue, emmenagogue, fungicide, hemostat, litholytic, stomachic, sedative, and vulnerary remedy (37).

Arial parts/leaves applications of $A$. eupatoria are given in Table 3 (33). It has been reported that giving Tisane (cold) to lambs has an antidiarrhoeal effect and Tisane (hot) has laxative effects in lambs (38).

\subsection{Recommendations on safety}

It should be used with caution when there is a constipation $(21,33)$. Side effects of nausea and constipation with excessive doses are likely (21).

\section{Phytochemistry}

The Agrimony plant contains tannins, volatile oil and coumarins (1), gum, a phytosterol (24), polysaccharides,

Table 2. Microscopic characters of Agrimonia eupatoria

\begin{tabular}{lll}
\hline & Diploid $(2 n=2 x=28)$ & \\
Chromosomes & Tetraploid $(2 n=4 x=56)$ & Perfectly normal, high pollen fertility (94-100\%) \\
Counts $(X=14)$ & Pentaploid $(2 n=5 x=70)$ & Abnormal meiotic course, high pollen sterility (74-88\%). Did not produce seeds \\
& Hexaploid $(2 n=6 x=84)$ & Perfectly normal, high pollen fertility (94-100\%) \\
\hline
\end{tabular}


Table 3. Applications of Agrimonia eupatoria

\begin{tabular}{|c|c|c|c|}
\hline Application & & Method & For \\
\hline \multirow{4}{*}{ Infusion } & & $\begin{array}{l}\text { A gentle remedy, especially in infants and children. Can be taken by } \\
\text { breastfeeding mothers. }\end{array}$ & Diarrhea \\
\hline & Eyewash & A weak infusion ( $10 \mathrm{~g}$ herb to $500 \mathrm{~mL}$ water) & Conjunctivitis \\
\hline & \multirow{2}{*}{ Gargle } & \multirow{2}{*}{ Use the infusion } & Sore throats \\
\hline & & & Nasal catarrh \\
\hline \multirow{4}{*}{ Tincture } & & \multirow{4}{*}{ More potent and drying than the infusion, and effective } & Condition involves excess phlegm or mucus \\
\hline & & & Cystitis urinary infections \\
\hline & & & Bronchitis \\
\hline & & & Heavy menstrual bleeding \\
\hline Poultice & & Of the leaves & Migraines \\
\hline \multirow{4}{*}{ Wash } & & & Wounds \\
\hline & & & Sores \\
\hline & & & Eczema \\
\hline & & & Varicose ulcers \\
\hline
\end{tabular}

and flavonoids such as luteolin. From its dried aerial parts, the polyphenolic-polysaccharide complex was isolated with $55 \times 10^{3} \mathrm{~g} / \mathrm{mol}$ molecular weight. It consisted mainly of pectin-like polysaccharides and polyphenolic moieties, composed of lignin-related units, with the dominance of dimethoxy phenyl structures. Agrimonia complex specific carbohydrate composition rich in highly esterified galacturonic acid, constituting thus highly methylated pectin network in which, besides arabinogalactan type II, the highly esterified homogalacturonan and rhamnogalacturonan type I are present, while some units being partially methylated (39). Investigated samples have been shown to contain about $8.2-10.9 \mathrm{mg} / \mathrm{g}$ of various flavonoids, $6.3-10.9 \mathrm{mg} / \mathrm{g}$ of various tannins (mostly agrimoniin, $2.6-5.4 \mathrm{mg} / \mathrm{g}$ ), and $0.6-0.9 \mathrm{mg} / \mathrm{g}$ of phenolic acids (40). The value of flavonoids determined in the leaves of agrimony (Agrimonia eupatoria) was $1.05 \mathrm{RU}$ (41). Total phenolic compounds of more than $19.61 \mathrm{mg}$ galic acid equivalent (GAE)/g to $220.31 \mathrm{mg}$ GA (gallic acid)/g, flavonoids of $20.58 \mathrm{mg} \mathrm{RU}$ (rutin)/g to $97.06 \mathrm{mg}$ $\mathrm{RU} / \mathrm{g}$, total tannins of $3.06 \mathrm{mg} \mathrm{GA} / \mathrm{g}$ to $207.27 \mathrm{mg} \mathrm{GA} / \mathrm{g}$, and pro-anthocyanidins of $4.15 \mathrm{CChE}$ (Cyanidin chloride equivalent)/g to $103.72 \mathrm{CChE} / \mathrm{g}$ have been identified (42), claiming that phenol compounds were the major group of constituents of this plant (40).
The primary metabolites

From the primary metabolites, the amino acid composition has been determined. Seventeen amino acids and their respective amounts were identified in A. eupatoria, which is shown in Table 4 (43).

The second metabolites

Sixty-eight out of 87 separated constituents of the volatile oil were quantified, which were more than $87.03 \%$ of the total contents. Isolated compounds from the leaf and root of $A$. eupatoria are shown in Table 5, isolated compounds from the leaf of A. eupatoria are shown in Table 6 (44), and the phenolic and flavonoid compounds isolated from free or glycosides of the leaf of A. eupatoria are shown in Table 7 .

Antioxidant potential and scavenging activity Agrimony's anti-inflammatory ability may be clarified by its antioxidant activity. The plant has polyphenolic compounds capable of activating endogenous antioxidant defense mechanisms (51). The tests done on A. eupatoria extract and its polyphenol-enriched fractions against reactive species are shown in Table 8 .

Table 4. Amino acid content of Agrimonia eupatoria herb

\begin{tabular}{|c|c|c|c|c|c|c|}
\hline Substance & General formula & $\begin{array}{l}\text { Content, } \mathrm{mg} / 100 \mathrm{mg} \\
\text { of the raw material }\end{array}$ & No. & Substance & General formula & $\begin{array}{c}\text { Content, } \mathrm{mg} / 100 \mathrm{mg} \text { of } \\
\text { the raw material }\end{array}$ \\
\hline Aspartic acid & $\mathrm{C} 4 \mathrm{H} 6 \mathrm{O} 4 \mathrm{~N}$ & 0.93 & 10 & Methionine & $\mathrm{C} 5 \mathrm{H} 10 \mathrm{O} 2 \mathrm{NS}$ & 0.31 \\
\hline Threonine & $\mathrm{C} 4 \mathrm{H} 9 \mathrm{O} 2 \mathrm{~N}$ & 0.39 & 11 & Isoleucine & $\mathrm{C} 6 \mathrm{H} 13 \mathrm{O} 2 \mathrm{~N}$ & 0.46 \\
\hline Serine & $\mathrm{C} 3 \mathrm{H} 7 \mathrm{O} 3 \mathrm{~N}$ & 0.62 & 12 & Leucine & $\mathrm{C} 6 \mathrm{H} 13 \mathrm{O} 2 \mathrm{~N}$ & 0.46 \\
\hline Glutamic acid & $\mathrm{C} 5 \mathrm{H} 8 \mathrm{O} 4 \mathrm{~N}$ & 0.15 & 13 & Tyrosine & $\mathrm{C9H} 13 \mathrm{O} 3 \mathrm{~N}$ & 0.23 \\
\hline Proline & $\mathrm{C} 5 \mathrm{H} 9 \mathrm{O} 2 \mathrm{~N}$ & 0.47 & 14 & Phenylalanine & $\mathrm{C} 9 \mathrm{H} 12 \mathrm{O} 2 \mathrm{~N}$ & 0.33 \\
\hline Cysteine & $\mathrm{C} 6 \mathrm{H} 12 \mathrm{~N} 2 \mathrm{O} 4 \mathrm{~S} 2$ & 0.08 & 15 & Histidine & $\mathrm{C} 6 \mathrm{H} 1102 \mathrm{~N} 3$ & 0.23 \\
\hline Glycine & $\mathrm{C} 2 \mathrm{H} 5 \mathrm{O} 2 \mathrm{~N}$ & 0.93 & 16 & Lysine & $\mathrm{C} 6 \mathrm{H} 13 \mathrm{O} 2 \mathrm{~N} 2$ & 0.53 \\
\hline Alanine & $\mathrm{C} 3 \mathrm{H} 7 \mathrm{O} 2 \mathrm{~N}$ & 0.69 & 17 & Arginine & $\mathrm{C} 6 \mathrm{H} 15 \mathrm{O} 2 \mathrm{~N} 4$ & 0.15 \\
\hline Valine & $\mathrm{C} 5 \mathrm{H} 11 \mathrm{O} 2 \mathrm{~N}$ & 0.69 & & & & \\
\hline
\end{tabular}


Table 5. The compounds isolated from the leaf and root essence of Agrimonia eupatoria

\begin{tabular}{|c|c|c|c|c|c|}
\hline No. & Compound Name & Molecule structure & No. & Compound Name & Molecule structure \\
\hline 1 & 1-(2-Furyl)-1-hexanone & $\mathrm{C} 10 \mathrm{H} 14 \mathrm{O} 2$ & 35 & Geraniol acetate & $\mathrm{C} 12 \mathrm{H} 20 \mathrm{O} 2$ \\
\hline 2 & 2,4-Dimethylbenzaldehyde & $\mathrm{C} 9 \mathrm{H} 100$ & 36 & Geranyl acetone & $\mathrm{C} 13 \mathrm{H} 22 \mathrm{O}$ \\
\hline 3 & 2-Cyclopropylidene-1,7,7-trimethyl-bicyolo $[2,2,1]$ heptane & $\mathrm{C} 13 \mathrm{H} 20$ & 37 & Hexanal & $\mathrm{C} 6 \mathrm{H} 12 \mathrm{O}$ \\
\hline 4 & 2-Methyl-4-hydroxyacetophenone & $\mathrm{C} 9 \mathrm{H} 2 \mathrm{OO} 2$ & 38 & L-Camphor & $\mathrm{C} 10 \mathrm{H} 160$ \\
\hline 5 & 3,4-Dimethylbenzaldehyde & $\mathrm{C} 9 \mathrm{H} 100$ & 39 & Linalool & $\mathrm{C} 10 \mathrm{H} 180$ \\
\hline 6 & 3-Octanol & $\mathrm{C} 10 \mathrm{H} 180$ & 40 & Longofolene & $\mathrm{C} 15 \mathrm{H} 24$ \\
\hline 7 & 4-Terpineol & $\mathrm{C} 10 \mathrm{H} 180$ & 41 & Muurolol & $\mathrm{C} 15 \mathrm{H} 26 \mathrm{O}$ \\
\hline 8 & 6,10,14-Trimethyl-2-pentadecanone & $\mathrm{C} 18 \mathrm{H} 360$ & 42 & Myristicin & $\mathrm{C} 11 \mathrm{H} 12 \mathrm{O} 3$ \\
\hline 9 & Acoradiene & $\mathrm{C} 15 \mathrm{H} 24$ & 43 & Neryl acetate & $\mathrm{C} 12 \mathrm{H} 20 \mathrm{O} 2$ \\
\hline 10 & Anethole & $\mathrm{C} 10 \mathrm{H} 12 \mathrm{O}$ & 44 & Nonanoic acid & $\mathrm{C} 9 \mathrm{H} 18 \mathrm{O} 2$ \\
\hline 11 & Aromadendrene & $\mathrm{C} 15 \mathrm{H} 24$ & 45 & Patchoulol & $\mathrm{C} 15 \mathrm{H} 26 \mathrm{O}$ \\
\hline 12 & Bergamot oil & $\mathrm{C} 12 \mathrm{H} 20 \mathrm{O} 2$ & 46 & P-Menth-1-en-4-ol & $\mathrm{C} 10 \mathrm{H} 180$ \\
\hline 13 & Borneol & $\mathrm{C} 10 \mathrm{H} 180$ & 47 & Pulegone & $\mathrm{C} 10 \mathrm{H} 160$ \\
\hline 14 & Bornyl acetate & $\mathrm{C} 12 \mathrm{H} 20 \mathrm{O} 2$ & 48 & Thymol & $\mathrm{C} 10 \mathrm{H} 140$ \\
\hline 15 & Camphene & $\mathrm{C} 10 \mathrm{H} 16$ & 49 & Torreyol & $\mathrm{C} 15 \mathrm{H} 26 \mathrm{O}$ \\
\hline 16 & Carvacrol & $\mathrm{C} 10 \mathrm{H} 140$ & 50 & Trans-Nerolidol & $\mathrm{C} 15 \mathrm{H} 26 \mathrm{O}$ \\
\hline 17 & Caryophyllene & $\mathrm{C} 15 \mathrm{H} 24$ & 51 & $\alpha$-Bisabolene & $\mathrm{C} 15 \mathrm{H} 24$ \\
\hline 18 & Caryophyllene oxide & $\mathrm{C} 15 \mathrm{H} 24 \mathrm{O}$ & 52 & $\alpha$-Cadinol & $\mathrm{C} 15 \mathrm{H} 26 \mathrm{O}$ \\
\hline 19 & Cedrol & $\mathrm{C} 15 \mathrm{H} 26 \mathrm{O}$ & 53 & $\alpha$-Campholenal & $\mathrm{C} 10 \mathrm{H} 160$ \\
\hline 20 & Cedryl acetate & $\mathrm{C} 17 \mathrm{H} 28 \mathrm{O} 2$ & 54 & $\alpha$-Cedrene & $\mathrm{C} 15 \mathrm{H} 24$ \\
\hline 21 & Copaene & $\mathrm{C} 15 \mathrm{H} 24$ & 55 & $\alpha$-Eudesmol & $\mathrm{C} 15 \mathrm{H} 26 \mathrm{O}$ \\
\hline 22 & Cubenol & $\mathrm{C} 15 \mathrm{H} 26 \mathrm{O}$ & 56 & $\alpha$-Guaiene & $\mathrm{C} 15 \mathrm{H} 24$ \\
\hline 23 & Cuparene & $\mathrm{C} 15 \mathrm{H} 24$ & 57 & $\alpha$-Himachalene & $\mathrm{C} 15 \mathrm{H} 24$ \\
\hline 24 & Curcumene & $\mathrm{C} 15 \mathrm{H} 22$ & 58 & $\alpha$-Longipinene & $\mathrm{C} 15 \mathrm{H} 24$ \\
\hline 25 & Cymene & $\mathrm{C} 10 \mathrm{H} 14$ & 59 & $\alpha$-Pinene & $\mathrm{C} 10 \mathrm{H} 16$ \\
\hline 26 & Decanoic acid & $\mathrm{C} 10 \mathrm{H} 20 \mathrm{O} 2$ & 60 & $\alpha$-Selinene & $\mathrm{C} 15 \mathrm{H} 24$ \\
\hline 27 & D-Limonene & $\mathrm{C} 10 \mathrm{H} 16$ & 61 & $\alpha$-Terpineol & $\mathrm{C} 10 \mathrm{H} 180$ \\
\hline 28 & E-Cadinene & $\mathrm{C} 15 \mathrm{H} 24$ & 62 & $\alpha$-trans-Ocimene & $\mathrm{C} 10 \mathrm{H} 16$ \\
\hline 29 & Epi-Cedrol & $\mathrm{C} 15 \mathrm{H} 26 \mathrm{O}$ & 63 & $\beta$-Cedrene & $\mathrm{C} 15 \mathrm{H} 24$ \\
\hline 30 & Epiglobulol & $\mathrm{C} 15 \mathrm{H} 26 \mathrm{O}$ & 64 & $\beta$-Pinene & $\mathrm{C} 10 \mathrm{H} 16$ \\
\hline 31 & Eucalyptol & $\mathrm{C} 10 \mathrm{H} 180$ & 65 & $\beta$-Selinene & $\mathrm{C} 15 \mathrm{H} 24$ \\
\hline 32 & Eugenol methyl ether & $\mathrm{C} 11 \mathrm{H} 14 \mathrm{O} 2$ & 66 & $\delta$-Cadinene & $\mathrm{C} 15 \mathrm{H} 24$ \\
\hline 33 & Farnesyl acetate & $\mathrm{C} 17 \mathrm{H} 28 \mathrm{O} 2$ & 67 & $\delta$-Guaiene & $\mathrm{C} 15 \mathrm{H} 24$ \\
\hline 34 & Furan,2,5-dibutyl- & $\mathrm{C} 12 \mathrm{H} 200$ & 68 & $\tau$-Cadinene & $\mathrm{C} 15 \mathrm{H} 24$ \\
\hline
\end{tabular}

Table 6. The compounds isolated from the leaf essence of Agrimonia eupatoria

\begin{tabular}{|c|c|c|c|c|c|}
\hline No. & Compound Name & Molecule structure & No. & Compound Name & Molecule structure \\
\hline 1 & 1-Hexanol & $\mathrm{C} 6 \mathrm{H} 14 \mathrm{O}$ & 8 & Germacrene D & $\mathrm{C} 15 \mathrm{H} 24$ \\
\hline 2 & 4,4-Dimethyladamantan-2-ol & $\mathrm{C} 12 \mathrm{H} 2 \mathrm{OO}$ & 9 & Hexahydrofarnesyl acetone & $\mathrm{C} 18 \mathrm{H} 360$ \\
\hline 3 & 4-Hydroxy-3-methylacetophenon & $\mathrm{C} 9 \mathrm{H} 10 \mathrm{O} 2$ & 10 & Isomenthone & $\mathrm{C} 10 \mathrm{H} 180$ \\
\hline 4 & Cadala-1(10),3,8-triene & $\mathrm{C} 15 \mathrm{H} 22$ & 11 & Longipinocarvone & $\mathrm{C} 15 \mathrm{H} 280$ \\
\hline 5 & Carvone & $\mathrm{C} 10 \mathrm{H} 140$ & 12 & Phenmethyl acetate & $\mathrm{C} 9 \mathrm{H} 10 \mathrm{O} 2$ \\
\hline 6 & Cis-7-Tetradecen-1-ol & $\mathrm{C} 14 \mathrm{H} 28 \mathrm{O}$ & 13 & Prenal & $\mathrm{C} 5 \mathrm{H} 8 \mathrm{O}$ \\
\hline 7 & Costunolide & $\mathrm{C} 15 \mathrm{H} 20 \mathrm{O} 2$ & 14 & $\beta$-Damascone & $\mathrm{C} 13 \mathrm{H} 180$ \\
\hline
\end{tabular}


Table 7. The phenolic and flavonoid compounds isolated in a form of free or glycosidic from leaf of Agrimonia eupatoria

\begin{tabular}{|c|c|c|}
\hline No. & Compound Name & Reference \\
\hline 1 & 1-O-Caffeoylquinic acid & $(40)$ \\
\hline 2 & 3-O-Caffeoylquinic acid & $(40)$ \\
\hline 3 & 3-O-p-coumaroylquinic acid & $(25)$ \\
\hline 4 & 3-O-p-Coumaroylquinic acid & $(40)$ \\
\hline 5 & 4-O-caffeoylquinic acid & $(25,40)$ \\
\hline 6 & 5-caffeoyl quinic acid & $(46)$ \\
\hline 7 & 5-O-caffeoylquinic acid (chlorogenic acid)b & $(25,40)$ \\
\hline 8 & Agrimoniin & $(25,40,46)$ \\
\hline 9 & Apigenin 7-O-glucoside (apigetrin) & $(25,40,46)$ \\
\hline 10 & Apigenin 7-O ß-D-glucuronide & $(25,40,45,47)$ \\
\hline 11 & Apigenin derivative & $(45)$ \\
\hline 12 & Apigenin O-glucuronide & $(46)$ \\
\hline 13 & Astragalin (Kaempferol 3-O-glucoside) & $(25,40,46-49)$ \\
\hline 14 & Caffeoyl-hexoside & $(45)$ \\
\hline 15 & Catechin & $(25,40,45,46,48)$ \\
\hline 16 & Ellagic acid & $(46)$ \\
\hline 17 & Hexahydroxydiphenoyl-glucose & $(50)$ \\
\hline 18 & Isovitexin= Apigenin 6-C-glucoside & $(25,40,46,48)$ \\
\hline 19 & Kaempferide & (49) \\
\hline 20 & Kaempferide 3-rhamnoside & (49) \\
\hline 21 & Kaempferide O-rhamnoside & $(46)$ \\
\hline 22 & Kaempferol & (49) \\
\hline 23 & Kaempferol 3- O- (6"- O- p- coumaroyl)-glucoside, (tiliroside) & $(46-48)$ \\
\hline 24 & Kaempferol 3- O- beta- D- (2"- O- acetyl- 6"- (E)- p- coumaroyl)- glucopyranoside (2"-acetyl- tiliroside) & (47) \\
\hline 25 & Kaempferol 3- O- ß- D- (2"- O-acetyl) glucopyranoside & $(47)$ \\
\hline 26 & Kaempferol 3-rhamnoside & (49) \\
\hline 27 & Kaempferol 3-rutinoside & (49) \\
\hline 28 & Kaempferol hexoside & $(25,40)$ \\
\hline 29 & Kaempferol malonylhexoside & $(25,40)$ \\
\hline 30 & Kaempferol O-acetylhexosyl-rhamnoside & (46) \\
\hline 31 & Kaempferol Oacetyl-hexosyl-O-rhamnoside & (46) \\
\hline 32 & Kaempferol O-(coumaroyl)-hexoside & $(50)$ \\
\hline 33 & Kaempferol O-malonylhexoside & $(46)$ \\
\hline 34 & Kaempferol-p-coumaroyl-hexoside & $(45)$ \\
\hline 35 & Keampferol 3-O-rutinosid & $(50)$ \\
\hline 36 & Luteolin 7-O-glucoside (cynaroside) & $(25,40,46)$ \\
\hline 37 & Luteolin 7-O-ß-D-glucopyranoside & (47) \\
\hline 38 & Luteolin 7-O-ß-D-glucuronide & $(25,40,45,47)$ \\
\hline 39 & Luteolin glucuronide isomer & $(25,40)$ \\
\hline 40 & Luteolin malonylhexoside & $(25)$ \\
\hline 41 & Luteolin-acetyl-hexoside & $(45)$ \\
\hline 42 & P-Coumaric acid & $(45,46,48)$ \\
\hline 43 & p-Coumaroil quinic acid & $(45)$ \\
\hline 44 & p-Coumaroyl acid hexoside & $(40)$ \\
\hline 45 & Procyanidin B-1 & $(45)$ \\
\hline 46 & Procyanidin B3= Procyanidin dimer B3= catechin $-(4 \beta \rightarrow 8)$-catechin (B3) & $(25,40,45,48)$ \\
\hline 47 & Procyanidin dimer B1= epicatechin- $(4 \beta \rightarrow 8)$ - catechin (B1) & (48) \\
\hline 48 & Procyanidin dimer B2= epicatechin- $(4 \beta \rightarrow 8)$-epicatechin (B2) & $(48)$ \\
\hline 49 & Procyanidin dimer $B 6=$ catechin $-(4 \beta \rightarrow 6)$ catechin $(B 6)$ & (48) \\
\hline 50 & Procyanidin dimer $B 7=$ epicatechin- $(4 \beta \rightarrow 6)$-catechin $(B 7)$ & (48) \\
\hline 51 & Procyanidin tetramer & $(46,48)$ \\
\hline
\end{tabular}


Table 7. Continued

\begin{tabular}{|c|c|c|}
\hline No. & Compound Name & Reference \\
\hline 52 & Procyanidin tetramer-B & (45) \\
\hline 53 & Procyanidin trimer & $(25,40,46,48)$ \\
\hline 54 & Procyanidin trimer $\mathrm{C} 1=$ epicatechin- $(4 \rightarrow 8)$-epicatechin- $(4 \rightarrow 8)$-epicatechin $(\mathrm{C} 1)$ & $(48)$ \\
\hline 55 & Procyanidin trimer $\mathrm{C} 2=$ catechin- $(4 \rightarrow 8)$-catechin- $(4 \rightarrow 8)$ - catechin $(\mathrm{C} 2)$ & (48) \\
\hline 56 & Procyanidin trimer EEC $=$ epicatechin- $(4 \rightarrow 8)$ - epicatechin- $(4 \rightarrow 8)$-catechin $(\mathrm{EEC})$ & $(48)$ \\
\hline 57 & Procyanidin-trimer-B & $(45)$ \\
\hline 58 & Protocatechuic acid & $(48)$ \\
\hline 59 & Quercetin 3-O-galactoside (hyperoside) & $(25,40,48)$ \\
\hline 60 & Quercetin 3-O-glucoside (isoquercitrin) (isoquercetin) & $(25,40,45-48)$ \\
\hline 61 & Quercetin 3-O-rhamnoglucoside (rutin) & $(25,40)$ \\
\hline 62 & Quercetin 3-O-rhamnoside & $(25,40,45)$ \\
\hline 63 & Quercetin 6_-O-galloyl-3-O-glucoside & $(40)$ \\
\hline 64 & Quercetin malonylhexoside isomer & $(25,40,46)$ \\
\hline 65 & Quercetin O-galloyl-hexoside & $(46)$ \\
\hline 66 & Quercetin rhamnoglucoside isomer & $(25,40)$ \\
\hline 67 & Quercetin-7-O-rhamnoside & $(45)$ \\
\hline 68 & Quercetin-acetyl-hexoside & $(45)$ \\
\hline 69 & Quercitin-acetil-glucoside & $(45)$ \\
\hline 70 & Quercitrin & $(47)$ \\
\hline 71 & Quinic acid & $(45)$ \\
\hline 72 & Rutin & $(25,45,47)$ \\
\hline 73 & Vitexin (Apigenin 8-C-glucoside) & $(40,46)$ \\
\hline
\end{tabular}

Table 8. Ex vivo measurements of antioxidant activity of Agrimonia eupatoria

\begin{tabular}{|c|c|c|}
\hline Phytochemical test & Reagent & Result \\
\hline \multirow[t]{2}{*}{$\begin{array}{l}\text { Free radical } \\
\text { scavenging activity } \\
\text { or the antioxidant } \\
\text { activity }\end{array}$} & $\begin{array}{l}\text { DPPH (2,2-diphenyl-1- } \\
\text { picrylhydrazyl) method }\end{array}$ & $\begin{array}{l}\text { Indicating a general radical scavenging activity, with a dose-dependent ability }(9.1-97.5 \%) \\
(5,8) \text {, quite similar to ascorbic acid }\left(\mathrm{EC}_{50}=17 \mu \mathrm{g}\right) \text { and much higher than Catechin }\left(\mathrm{EC}_{50}=140\right. \\
\mu \mathrm{g})(50) \text {. The } \mathrm{MeOH} \text { extract and two Sep-Pak fractions ( } 30 \text { and } 60 \%) \text { showed prominent } \\
\text { free radical scavenging activity. } \mathrm{RC}_{50} \mathrm{~s} \text { were determined as } 4.64 \times 10^{-4}, 5.13 \times 10^{-3} \text { and } 4.73 \times 10^{-} \\
{ }^{4} \mathrm{mg} / \mathrm{mL}(4) \text {. The antioxidant activity was highest for the acetone extract and ranged from } \\
97.13 \% \text { to } 27.73 \%(42) \text {. }\end{array}$ \\
\hline & Reducing power & $\begin{array}{l}\text { Extracts of } A \text {. eupatoria showed moderate reducing power compared with the positive } \\
\text { control, and showed activity in all examined concentrations. Hence, concluded that } \\
\text { reducing power depends on concentration (42). }\end{array}$ \\
\hline \multirow{2}{*}{$\begin{array}{l}\text { Superoxide anion } \\
\text { scavenging effect }\end{array}$} & $\begin{array}{l}\text { Detected by Nitro Blue } \\
\text { Tetrazolium (NBT) reduction }\end{array}$ & Inhibited the NBT reduction in a dose-dependent manner \\
\hline & $\begin{array}{l}\text { Peroxyl and hydroxyl } \\
\text { radicals }\end{array}$ & A very good scavenging capacity. The extract is an efficient scavenger of peroxyl radicals. \\
\hline \multirow{4}{*}{ Oxidant species } & $\begin{array}{l}\text { Hydrogen peroxide }\left(\mathrm{H}_{2} \mathrm{O}_{2}\right) \\
\text { assay }\end{array}$ & $\begin{array}{l}\text { Exhibited } \mathrm{H}_{2} \mathrm{O}_{2} \text { scavenging activity ( } 8 \text { ). Agrimony extract (AE) decreased both the superoxide } \\
\text { dismutase (SOD) and catalase (CAT) expressions (45). }\end{array}$ \\
\hline & $\begin{array}{l}\text { Hypochlorous acid (Elastase } \\
\left(\text { E) }+\alpha_{1} \text {-antitrypsin }\left(\alpha_{1}\right)+\right. \\
\text { Hypochlorous acid (HOCl)) } \\
\text { and inhibition of Elastase }\end{array}$ & $\begin{array}{l}\text { Extract abolished elastase activity; affected the } \alpha_{1} \text { - antitrypsin activity, but promoted some } \\
\text { inhibition of Elastase activity. }\end{array}$ \\
\hline & \multirow{2}{*}{ Peroxynitrite } & Inhibited the dihydrorhodamine 123 oxidation (8) \\
\hline & & S-nitroso-N-acetylpenicillamine (SNAP) assays (46) \\
\hline \multicolumn{2}{|c|}{$\begin{array}{l}\text { 2,2'-azinobis-(3-ethylbenzothiazoline-6-sulfonic } \\
\text { acid) (ABTS) (+) radical decolourisation reaction } \\
\text { system }\end{array}$} & 6.7-79.5\% $(5,53)$ and $\mathrm{RC}_{50 \mathrm{~s}}$ were determined as Agrimony $=0.79 \mathrm{mg} / \mathrm{mL}(45)$. \\
\hline \multicolumn{2}{|c|}{ Trolox equivalent antioxidant capacity (TEAC) } & $\begin{array}{l}\text { Showed the scavenging activities (48), (TEAC 3.76+/-0.5mM/QE 702.29+/-6.82 microM) } \\
\text { (6). }\end{array}$ \\
\hline \multicolumn{2}{|c|}{$\begin{array}{l}\text { Thiobarbituric acid reactive substances (TBARS) } \\
\text { method }\end{array}$} & The lipid peroxidation inhibition (48), 104.8 \pm 11.4 (\% control) (53) \\
\hline
\end{tabular}


Table 8. Continued

\begin{tabular}{|c|c|c|}
\hline Phytochemical test & Reagent & Result \\
\hline The phenolic content & & $\begin{array}{l}\text { The relevant content of phenols and significant antioxidant activities were observed for the } \\
\text { aqueous-alcoholic extract (49). Ascertained by High-performance liquid chromatography- } \\
\text { diode array detection (HPLC-DAD) (55) ranged from } 19.61 \mathrm{mgGA} / \mathrm{g} \text { to } 220.31 \mathrm{mgGA} / \mathrm{g}(43) \text {. } \\
\text { Achieved by HPLC-PDA-ESI/MS }{ }^{n} \text { (46). }\end{array}$ \\
\hline \multicolumn{2}{|c|}{$\begin{array}{l}\text { The effect of in vitro digestion upon Caco- } 2 \text { cells via } \\
\text { LC-MS/MS }\end{array}$} & $\begin{array}{l}\text { 3,4,5-Trihydroxybenzaldehyde was stable throughout the digestive process, whereas } \\
\text { Chlorogenic acid remained stable after the stomach phase, Rutin was decreased after the } \\
\text { stomach phase (52). }\end{array}$ \\
\hline \multicolumn{2}{|c|}{ Capacity to inhibit lipid peroxidation } & Reacted \\
\hline $\begin{array}{l}\text { Xanthine oxidase } \\
\text { activity }\end{array}$ & Uric acid formation & A dose-dependent inhibition (8) \\
\hline Carbonyl content & Protein oxidation & $86.8 \pm 3.7$ (\% control) \\
\hline 8-ohdg & DNA oxidation & $149.6 \pm 34.0$ (\% control) (53) \\
\hline Oxidative damage & Plasmid DNA & $\begin{array}{l}\text { Agrimony extract possesses better antioxidant properties to compare with rutin as } \\
\text { standard. }\end{array}$ \\
\hline Cytotoxicity test & $\begin{array}{l}24 \mathrm{~h} \text { incubation on THP-1 } \\
\text { cell line }\end{array}$ & No cytotoxic effect was observed. \\
\hline Skin flap viability & $\begin{array}{l}\text { On male Sprague-Dawley } \\
\text { rats }\end{array}$ & A. eupatoria extract has the capacity to act on damaged skin (45). \\
\hline
\end{tabular}

$\mathrm{EC}_{50}$ : micrograms of freeze-dried samples required to scavenge $50 \%$ of the DPPH. Phytochemical tests were done on $A$. eupatoria extracts and its fractions.

Table 9. Used extraction techniques for Isoflavonoid's isolation and determination

\begin{tabular}{llll}
\hline Extraction technique & Diadzein & Genistein & Biochanin \\
\hline Supercritical fluid extraction (SFE) & $19.7 \pm 0.8$ & N.d. & $1.3 \pm 0.1$ \\
Pressurized fluid extraction & $4.8 \pm 0.3$ & N.d. & $0.6 \pm 0.1$ \\
Matrix solid phase dispersion & $2.8 \pm 0.4$ & $0.2 \pm 0.1$ & N.d \\
Ultrasonic extraction in an ultrasonic bath (USE) & $5.7 \pm 0.1$ & N.d. & N.d. \\
Ultrasonic homogeniser (HOM) & $12.8 \pm 0.1$ & N.d. & 0.1 \\
Soxhlet apparatus (SOX) & N.d. & $0.9 \pm 0.2$ & \\
\hline
\end{tabular}

N.d., concentration below the limit of detection.

Extraction techniques

Various extraction techniques have been used for the isolation and determination of A. eupatoria's Isoflavonoids. The techniques are listed in Table 9 (54).

Pharmacological activities

Agrimonia eupatoria's physio-pharmacological activities are shown in Table 10.

\section{Toxicity}

The safety and efficacy of $A$. eupatoria have been confirmed during a very long period of its traditional application. Recent studies have shown that A. eupatoria aqueous extract (AEE) consumption by subjects was safe and generally well-tolerated without severe adverse events (65). Agrimony is an herbal medicine, which its safety is comparable with coffee (37). But, since Agrimony is an indication of geogenic contamination of flysch soils, elements like $\mathrm{Ni}, \mathrm{Co}, \mathrm{Mn}, \mathrm{Cu}, \mathrm{Cr}, \mathrm{V}$, and Mo typically concentrate in the roots and shoots of the plant. So, high uptake of these elements by Agrimony growing on similar soils is possible (69).

\section{Conclusion}

This review presents A. eupatoria description, history, and advances in phytochemistry and other aspects. Pharmacological studies carried out on its extracts and traditional uses revealed that it could be an important source for new drugs.

\section{Authors' contributions}

MGP and NM conceived of the presented idea; MGP developed the article, performed the computations, wrote, and prepared the manuscript; NM encouraged; while author NM and SHM supervised the research and critical revision of the article. MRK and MRM made the final version. All authors read the manuscript and confirmed the publication of the final version.

\section{Conflict of interests}

There is no conflict of interest

\section{Ethical considerations}

Ethical issues including text plagiarism, misconduct, manipulation or appropriation, data fabrication, 
Physio-pharmacological

activities of Agrimonia Plant part/extract Dose/model

Worked on

For the aqueous extract single-dose of

Aerial parts/Aqueous $199.18 \mathrm{mg} / \mathrm{kg}$ (mice) and $99.59 \mathrm{mg} /$

extract + extraction $\mathrm{kg}$ (rats); for the fraction, single dose

with ethyl acetate of $36.24 \mathrm{mg} / \mathrm{kg}$ (mice) and $18.12 \mathrm{mg} /$

$\mathrm{kg}$ (rats)

The mouse carrageenan-induced

paw edema model for in vitro anti-

inflammatory activity (rats received

Analgesic and anti-

inflammatory

Arial parts/water

infusion and ethy

$99.59 \mathrm{mg} / \mathrm{kg}$, and $199.18 \mathrm{mg} / \mathrm{kg}$ doses

of infusion and $18.12 \mathrm{mg} / \mathrm{kg}$, and 36.24

$\mathrm{mg} / \mathrm{kg}$ for the fraction). The acetic acid-

induced writhing and hot-plate tests

for peripheral and central analgesic

potential (mice received $199.18 \mathrm{mg}$ /

$\mathrm{kg}$, and $398.26 \mathrm{mg} / \mathrm{kg}$, for infusion and

$36.24 \mathrm{mg} / \mathrm{kg}$, and $72.48 \mathrm{mg} / \mathrm{kg}$ for the

fraction) and formalin assay to assess

both activities (mice received 72.48

$\mathrm{mg} / \mathrm{kg}$ of the fraction).

Antiadhesive activity

Ethanol extract

Against Campylobacter jejuni

Campylobacter jejuni

Hydro ethanol extract Modified microdilution assay

Helicobacter pylori and Campylobacter jejuni

n-Hexane, DCM and MeOH extracts and Bacillus cereus, Bacillus subtilis, Escherichia coll,

four Sep-Pak fractions of the $\mathrm{MeOH}$ Pseudomonas aeruginosa, Salmonella goldcoast, and

extract.

Staphylococcus aureus

Staphylococcus aureus, Pseudomonas aeruginosa, and Escherichia coli

P. mirabilis and $P$. aeruginosa

Aerial parts/ethano

diethyl ether, water,

and acetone extracts

Aerial flowering

parts/Aqueous

extracts
Standard drug Result

The anti-inflammatory and peripheral analgesic properties of agrimony were confirmed. No signals of renal or hepatic toxicities detected (55).
Diclofenac

sodium and

morphine
In vivo anti-inflammatory and analgesic activities were verified (46)
No significant antiadhesion activity $\left(\mathrm{IC}_{(50)}\right.$ value $>35 \mathrm{mg} / \mathrm{mL}$ ) was found for Agrimonia eupatoria (56).

Agrimonia eupatoria was among the most active herbal extracts in inhibiting the growth of Helicobacter pylori (3).

The n-Hexane extract showed an inhibitory effect against Bacillus cereus and Bacillus subtilis. Sep-Pak fractions eluted with 30,60 , and $80 \% \mathrm{MeOH}$ in water showed significant antibacterial activity (4).
Fucidin ointment

Tetracycline
The ethanolic extract was more effective in inhibiting tested bacteria (57).

Biofilm inhibitory concentration required to reduce biofilm coverage by $50 \%$ values for acetone extract was $4315 \mathrm{mg} /$ $\mathrm{mL}$ for P. mirabilis and $4469.5 \mathrm{mg} / \mathrm{mL}$ for $P$. aeruginosa (42).

Displayed inhibition of butyrylcholinesterase (BuChE), acetylcholinesterase (AChE), and inhibition of cholinesterases by apigenin, luteolin, and quercetin glycosides (50). 
Table 10. Continued

\begin{tabular}{|c|c|c|c|c|}
\hline $\begin{array}{l}\text { Physio-pharmacological } \\
\text { activities of Agrimonia } \\
\text { eupatoria }\end{array}$ & Plant part/ extract & Dose/ Model & Worked on & Standard drug \\
\hline
\end{tabular}

\section{eupatoria}

Anticoagulant activity Dried aerial parts

man plasma-derived from healthy donors

\section{Into the diet $(62.5 \mathrm{~g} / \mathrm{kg})$ and drinking} water $(2.5 \mathrm{~g} / \mathrm{L}),(1 \mathrm{mg} / \mathrm{mL})$ on the

Aqueous extract abdominal muscle, $0.25-1 \mathrm{mg} / \mathrm{mL}$ on BRIN-BD11 cells/Streptozotocin (STZ)diabetic

Anti-diabetic

Water infusion

Streptozotocin-induced diabetes mellitus (DM) rat model $/ 200 \mathrm{mg} / \mathrm{L}$

Supplied in the diet $(6.25 \%$ by weight) or as decoctions or infusions (1 mellitus

$\mathrm{g} / 400 \mathrm{~mL}$ ) in place of drinking water/

Streptozotocin (200 mg/kg i.p.) for 12

days

Hydro-alcoholic

extract and a

polyphenol-enriched microplate-based broth dilution assay

fraction
Mice, abdominal muscle, BRIN-BD11 pancreatic B-cell line

Male Wistar rats
Anti-inflammatory

Activity
Enzyme-linked immunosorbent assay,

nitric oxide assay, and Western blotting.
BV2 microglial cells
The A eupatoria complex prevents the development of plasma clots, primarily in the intrinsic blood coagulation cascade pathway. It is primarily an indirect inhibitor of thrombin, mediated by antithrombin or by heparin cofactor II (39)

The presence of antihyperglycemic, insulin-releasing and insulin-like activity in Agrimony eupatoria demonstrated (15)

In the avoidance and/or adjuvant treatment of developing cardiovascular problems linked to DM and sicknesses related to oxidative stress, $A$. eupatoria extract suggests its higher clinical potential (58).

Treatment with agrimony reduced the level of hyperglycemia during the development of streptozotocin diabetes but did not reduce the rate of body weight loss. Certain traditional plant treatment for diabetes, namely Agrimony (14).

Agrimonia eupatoria anti-inflammatory activity mechanism could be its significant scavenging capacity of reactive species by its polyphenols (8).

Agrimoniae herba suppressed lipopolysaccharideinduced nitric oxide production in BV2 microglial cells and lipopolysaccharide-induced production of proinflammatory cytokines such as interleukin 1 beta, tumor necrosis factor, and interleukin 6 in a dose-dependent manner had no cytotoxicity and inhibited the expression of inducible nitric oxide synthase. It may be used as a form of pharmaceutical acupuncture therapy in the treatment of brain inflammation (9).

Tea One-month Healthy humans
Has potential in improving markers of lipid metabolism and inflammation (7). 
Table 10. Continued

Physio-pharmacological

activities of Agrimonia Plant part/extract

Dose/model

Worked on

Standard drug Result

eupatoria

From 2 to $0.004 \mathrm{mg} / \mathrm{mL} / 96$ well plate Selected gram-positive Staphylococcus aureus and grammicrodilution method (200, 40, and 8 negative Pseudomonas aeruginosa and Escherichia coli $\mu \mathrm{g} / \mathrm{mL}$

Antimicrobial activity

Extracts

Aerial parts/ Hot water, aqueous extract

\section{Primary human skin fibroblasts (line} C688)

Aerial parts /ethanol, diethyl ether, water, and acetone extracts and acetone extracts demonstrated the highest activity

Antimicrobial and antifungal activity
The antimicrobial activity was tested by determining the minimum inhibitory concentration (MIC) using the microdilution method with resazurin bacteria of relevance in wounds

Escherichia coli ATCC 25922, E. coli O44, Vibrio cholerae O395-tacCTB strain, and Lactobacillus rhamnosus

24 microorganisms including 18 strains of bacteria (probiotics strains: Lactobacillus rhamnosus, Bacillus subtilis IP 5832, and Bifidobacterium animalis subsp. lactis; standard strains: Staphylococcus aureus ATCC 25923, Enterococcus faecalis

A Escherichia coli ATCC 25922, B. subtilis ATCC 6633 , and $P$. aeruginosa ATCC 27853; and clinical isolates S. aureus, E. faecalis, Bacillus cereus, B. subtilis, E. coli, Salmonella enterica, Salmonella typhimurium, Klebsiella pneumoniae, P. mirabilis, and $P$. aeruginosa) and six strain of fungi (Aspergillus niger, Aspergillus flavus, Penicillium chrysogenu m, Penicillium italicum, C. albicans ATCC 10231, and Candida albicans)

\section{Tetracycline,}

ampicillin,

amphotericin B

and itraconazole
Moderate activity for Potentilla reptans L (59).

Displayed modest bacteriostatic potentials. Suppressed the binding of cholera toxin subunit $B$ to the cell surface and immobilized $\mathrm{GM}_{1}$ ganglioside (60).

The strongest antimicrobial activity was detected on $G$ bacteria, especially on probiotic species. The acetone extract demonstrated the highest activity (42)
Anti-nociceptive effect Aerial part/ Ethanolic $200 \mathrm{mg} / \mathrm{kg} /$ cisplatin-induced extract neuropathic pain model
Male Sprague-Dawley rats
In the pin-prick test and plantar test, $A$. eupatoria extract displayed an antinociceptive property with a lower withdrawal time and a higher withdrawal threshold in the paw-withdrawal threshold test as compared to contro animals. In the case of cold-allodynia, increased pawwithdrawal duration in the chemical test, and showed superior activity to gabapentin. A. eupatoria extract found to possess therapeutic potential for the treatment of neuropathic pain (61) 
Table 10. Continued

Physio-pharmacological

activities of Agrimonia

eupatoria

\section{Plant part/ extract}

Dose/ Model

Worked on

Standard drug Result

Acetone, methanol,
aqueous, acetone-
hexane, acetone-t-
butyl methyl ether,
acetone-n- BuOH,
acetone-aqueous
extracts

In DPPH radical scavenging, ABTS(+)

radical decolorization reaction systems

Tea

Trolox equivalent antioxidant capacity

(TEAC)

Aerial flowerin

parts/Aqueous

extracts

Arial parts/water,

acetone, In DPPH radical scavenging and

diethyl ether extracts Reducing power

Arial parts/infusion and ethyl acetate fraction

DPPH, superoxide anion, hydroxy

radical, and SNAP assays
Healthy humans

Broiler chicken
Radical scavenging capacity of agrimony extracts varied in a wide range and depending on the polarity of the solvent used to obtain the extract (9.1-97.5\% in DPPH reaction and $6.7-79.5 \%$ in ABTS reaction) (5).

Honeybush,

rooibos, black

and green tea

As compared to the foreign plants examined, it can be considered a rich source of water-soluble antioxidants (6).

Antioxidant activity of Agrimonia extract can be affected especially by hexahydroxydiphenoyl (HHDP)-glucose and quercetin glycosides (50).

Higher concentrations of acetone extract and vitamin C operated in a similar way, confirming the high antioxidant activity of Agrimony. All tested extracts showed a concentration-dependent antiradical activity Reducing the power of acetone extract was found to be the most active, followed by ethanol, water, and diethyl ether extracts (acetone $>$ ethanol $>$ water $>$ diethyl ether) (42).

Showed a significant antiradical activity against all tested radicals. Decreased NO levels in vitro. Fraction being more active than infusion. They are potential sources of antiradical and anti-inflammatory polyphenols (46).

Has potential in improving markers oxidative status in healthy adults (7).

Can beneficially influence the antioxidant status of thigh meat thus improve meat quality (62).

Antioxidant status

$0.1 \% \& 0.2 \%$

agrimony extract

Supplied through drinking water

$0.2 \%$ agrimony extract Supplied with drinking water/(2:1000)
Clove (Syzygium

aromaticum L.) It has a potential to increase the antioxidant status (63). 
eupatoria

Anti-tumor

Aqueous and

methanol extracts

$6.0,12.0,24.0,48.0$ and $96.0 \mu \mathrm{g} / \mathrm{mL}$

Human cervical cancer; Hela and Rhabdomyosarcoma (RD);

$\mathrm{RD}$ cell lines and a primary cell culture; mouse embryo

fibroblast; MEF

\section{Aerial parts (stems}

Antiviral activity and leaves)/ Aqueous

extract

Hepatitis B virus (HBV)

Administered in the water at a final

concentration of $0.1 \%$ after 42 days of

Enzymatic and non- Extracts extract from feeding the level of reduced glutathione 120 one-day-old broilers COBB500

enzymatic antioxidants the crop tops

was measured in the plasma and in

liver, heart, and kidney mitochondria

Growth performance,

and selected indices of $\quad 0.2 \%$ agrimony extract Supplied with drinking water/ (2:1000) Broiler chickens at the age of 42 days

lipid profile

Hepatic oxidative stress. Aqueous extracts

(leaves)

For 4 weeks

Mice

$10,30,100$, and $300 \mathrm{mg} / \mathrm{kg} / \mathrm{d} /$ chronic

Water extract ethanol-induced liver injury

Hepatoprotective

effects

Aqueous extract
$(160 \mathrm{mg} / \mathrm{d}) /$ two capsules twice a day for 8 weeks
Subjects aged between 20 and 70 years who were diagnosed with mild to moderately elevated ALT levels (between 45 and $135 \mathrm{IU} / \mathrm{L})$.

Five plant extracts concentrations showed concentrationdependent anti-tumor properties, and the methanol extract reported better growth inhibition percentage (PGI) values than aqueous extract in Hela and RD cell lines, while MEF cells, reported lower PGI values. Among these concentrations, for the three examined timespans, $96.0 \mu \mathrm{g} /$ $\mathrm{mL}$ was the best in generating PGI in RD and Hela cancer cell lines (13).

The extract prepared at $60{ }^{\circ} \mathrm{C}$ has the greatest effect. The inhibitory activity was the highest at mid-July. Agrimonia genus plants contain potential antiviral activity against HBV (16).

The activity of superoxide dismutase had a significant decrease. The application of agrimony extract appears to be suitable for the antioxidant effect against peroxidation of gamma-linolenic acid (63).

Clove (Syzygium Clove (Syzygium powder

Water
It fails to influence either the selected lipid metabolism indices or the growth performance (64).

Led to a decrease in catalase activity produced a decrease in SOD activity. In general, agrimony appeared to be a promising extract, in protection; and was even slightly toxic (53).

Agrimonia eupatoria water extract enhanced chronic ethanol-induced liver damage, likely because of oxidative stress suppression and Toll-like receptor (TLR) -mediated inflammatory signals (10).

A significant reduction in elevated alanine transaminase (ALT) and serum triglyceride (TG) was observed (65). 
Table 10. Continued

Physio-pharmacological

activities of Agrimonia

eupatoria

Irritable bowel

syndrome (IBS)

Aerial parts

formula containing Agrimony

Worked on

Result

\section{Neuroprotective}

Methanolic extract

Glutamate-injured HT22 cells

Oxidative stability

\section{$0.1 \% \& 0.2 \%$}

agrimony extract

Supplied with drinking water

Oxidative stress

Aqueous-alcoholic extract.

$1 \%, 2.5 \%, 5 \%, 7.5 \%, 10 \%, 12.5 \%$ and $15 \% / 24 \mathrm{~h}$

Areal parts/aqueous

Wound healing activity extract and crude

\section{$10 \mathrm{mg} / \mathrm{mL}$}

ethanolic extract

Pulverized in

combination with

three more herbs

Agrimonia eupatoria

$\begin{array}{ll}\text { Wound healing scar } & \text { (A) \& Nelumbo } \\ \text { formation inhibition } & \text { nucifera Gaertn (N) }\end{array}$

\& Boswellia carterii

(B) and Pollen Typhae

angustifoliae $(\mathrm{P})$

(ANBP)
Broiler chickens

Cell culture model 3T3-L1 pre-adipocytes

Patients who fulfilled the Rome II criteria for irritable bowel

syndrome.

Staphylococcus aureus, Pseudomonas aeruginosa, and

Escherichia coli

New Zealand white rabbits

ANBP plays dual roles, promoting wound healing and

alleviating scar formation (68).

habit IBS or improving bowel habit in individuals with

diarrhea-predominant but it improved a number of IBS symptoms significantly (66).

Astragalin, isoquercitrin, quercitrin, and luteolin 7-O-B-Dglucuronide compounds showed neuroprotective effects on glutamate-induced toxicity in HT22 cells (47).

Can beneficially influence the oxidative stability of thigh meat thus improve meat quality (62).

Pretreatment of cells with extract significantly reduced the stimulatory effect of the oxidizing agent on gene expression (6).

The wound healing was completed in 10 days by using the

Fucidin ointment ethanolic extract ointment (57).

The DA-IBS formula was not effective in alternating bowe 
falsification, redundant publication as well as duplicate submissions have been carefully observed by authors.

\section{Funding/Support}

This research did not receive any specific grant from funding agencies in the public, commercial, or profit sectors. There has been no financial support for this work.

\section{References}

1. Chevallier A. The Encyclopedia of Medicinal Plants. London: Dorling Kindersley; 1996. p. 336.

2. GRIN Taxonomy for Plants, Taxon: Agrimonia eupatoria L. [Internet]. United States Department of Agriculture. 2014. Available from: http://www.ars-grin.gov/cgibin/npgs/html/ taxon.pl?1786.

3. Cwikla C, Schmidt K, Matthias A, Bone KM, Lehmann R, Tiralongo E. Investigations into the antibacterial activities of phytotherapeutics against Helicobacter pylori and Campylobacter jejuni. Phytother Res. 2010;24(5):649-56. doi: $10.1002 /$ ptr.2933.

4. Copland A, Nahar L, Tomlinson CT, Hamilton V, Middleton M, Kumarasamy Y, et al. Antibacterial and free radical scavenging activity of the seeds of Agrimonia eupatoria. Fitoterapia. 2003;74(1-2):133-5. doi: 10.1016/ s0367-326x(02)00317-9.

5. Venskutonis PR, Skemaite M, Ragazinskiene O. Radical scavenging capacity of Agrimonia eupatoria and Agrimonia procera. Fitoterapia. 2007;78(2):166-8. doi: 10.1016/j. fitote.2006.10.002.

6. Ivanova D, Gerova D, Chervenkov T, Yankova T. Polyphenols and antioxidant capacity of Bulgarian medicinal plants. J Ethnopharmacol. 2005;96(1-2):145-50. doi: 10.1016/j. jep.2004.08.033.

7. Ivanova D, Vankova D, Nashar M. Agrimonia eupatoria tea consumption in relation to markers of inflammation, oxidative status and lipid metabolism in healthy subjects. Arch Physiol Biochem. 2013;119(1):32-7. doi: 10.3109/13813455.2012.729844.

8. Correia HS, Batista MT, Dinis TC. The activity of an extract and fraction of Agrimonia eupatoria L. against reactive species. Biofactors. 2007;29(2-3):91-104. doi: 10.1002/ biof.552029209.

9. Bae H, Kim HJ, Shin M, Lee H, Yin CS, Ra J, et al. Inhibitory effect of Agrimoniae Herba on lipopolysaccharide-induced nitric oxide and proinflammatory cytokine production in BV2 microglial cells. Neurol Res. 2010;32 Suppl 1:53-7. doi: 10.1179/016164109x12537002794002.

10. Yoon SJ, Koh EJ, Kim CS, Zee OP, Kwak JH, Jeong WJ, et al. Agrimonia eupatoria protects against chronic ethanol-induced liver injury in rats. Food Chem Toxicol. 2012;50(7):2335-41. doi: 10.1016/j.fct.2012.04.005.

11. Coady Y, Boylan F. Ethnopharmacology in Ireland: an overview. Rev Bras Farmacogn. 2014;24(2):197-205. doi: 10.1016/j.bjp.2014.04.002.

12. Copland A, Nahar L, Tomlinson CT, Hamilton V, Middleton M, Kumarasamy Y, et al. Antibacterial and free radical scavenging activity of the seeds of Agrimonia eupatoria. Fitoterapia. 2003;74(1-2):133-5. doi: 10.1016/ s0367-326x(02)00317-9.

13. Ad'hiah AH, Al-Bederi ONH, Al-Sammarrae KW. Cytotoxic effects of Agrimonia eupatoria L. against cancer cell lines in vitro. J Assoc Arab Univ Basic Appl Sci. 2013;14(1):87-92. doi: 10.1016/j.jaubas.2013.01.003.

14. Swanston-Flatt SK, Day C, Bailey CJ, Flatt PR. Traditional plant treatments for diabetes. Studies in normal and streptozotocin diabetic mice. Diabetologia. 1990;33(8):4624. doi: $10.1007 / \mathrm{bf00405106.}$

15. Gray AM, Flatt PR. Actions of the traditional antidiabetic plant, Agrimony eupatoria (agrimony): effects on hyperglycaemia, cellular glucose metabolism and insulin secretion. Br J Nutr. 1998;80(1):109-14. doi: 10.1017/ s0007114598001834.

16. Kwon DH, Kwon HY, Kim HJ, Chang EJ, Kim MB, Yoon SK, et al. Inhibition of hepatitis B virus by an aqueous extract of Agrimonia eupatoria L. Phytother Res. 2005;19(4):355-8. doi: $10.1002 /$ ptr.1689.

17. Soltani A. Encyclopedia of Traditional Medicine (Dictionary of Medicinal Plants). Tehran: Iran University of Medical Sciences and Health Services; 2011. p. 1-3.

18. Jarmila E, Juraj C, Jana S. Medicinal Herbs in Folk Medicine and Magic from Slovak Region Kysuce versus Their Current Usage. American Journal of Ethnomedicine. 2015;2(1):6878.

19. Ghahreman A, Okhovvat AR. Matching the Old Medicinal Plant Names with Scientific Terminology. University of Tehran Press; 2004.

20. Zargari A. Medicinal Plants. Tehran: Institute of Tehran University Press; 1999. p. 19-24.

21. Tobyn G, Denham A, Whitelegg M. The Western Herbal Tradition E-Book: 2000 Years of Medicinal Plant Knowledge. Elsevier Health Sciences; 2010. doi:10.1016/ b978-0-443-10344-5.00011-2.

22. Ripka G. Eriophyoid mites (Acari: Prostigmata: Eriophyoidea) from Hungary: a new species on Agrimonia eupatoria (Rosaceae) and new record on Convolvulus arvensis (Convolvulaceae). Zootaxa. 2014;3900(2):263-70. doi: $\quad$ 10.11646/zootaxa.3900.2.6.

23. Anisimovas E, Diškus A, Stonis JR. First Survey of Nepticulidae (Insecta: Lepidoptera) in čepkeliai state nature reserve, Lithuania. Acta Zool Litu. 2006;16(3):221-8. doi: 10.1080/13921657.2006.10512735.

24. Keys JD. Chinese Herbs. Tuttle Publishing; 2011.

25. Granica S, Kluge H, Horn G, Matkowski A, Kiss AK. The phytochemical investigation of Agrimonia eupatoria L. and Agrimonia procera Wallr. as valid sources of Agrimoniae herba--The pharmacopoeial plant material. J Pharm Biomed Anal. 2015;114:272-9. doi: 10.1016/j.jpba.2015.05.027.

26. Sojneková M, Chytrý M. From arable land to species-rich semi-natural grasslands: succession in abandoned fields in a dry region of central Europe. Ecol Eng. 2015;77:373-81. doi: 10.1016/j.ecoleng.2015.01.042.

27. Ali K, Khan N, Rahman IU, Ahmad H, Jury S. Multivariate analysis and vegetation mapping of a biodiversity hotspot in the Hindu Kush Mountains. Int J Adv Res. 2015;3(6):9901006.

28. Dar JA, Sundarapandian S. Patterns of plant diversity in seven temperate forest types of Western Himalaya, India. J Asia Pac Biodivers. 2016;9(3):280-92. doi: 10.1016/j. japb.2016.03.018.

29. Mägi M, Semchenko M, Kalamees R, Zobel K. Limited phenotypic plasticity in range-edge populations: a comparison of co-occurring populations of two Agrimonia species with different geographical distributions. Plant Biol (Stuttg). 2011;13(1):177-84. doi: 10.1111/j.1438- 
8677.2010.00342.x

30. Najafpour Navaei M, Golipour M, Parsa E. The effects of densities and planting dates on seed yield of Agrimonia eupatoria L. Iranian Journal of Medicinal and Aromatic Plants. 2008;24(2):198-206.

31. Póvoa O, Monteiro A. Geographic Distribution and Propagation of Agrimonia eupatoria L. and Melissa officinalis L. from Portugal. I International Medicinal and Aromatic Plants Conference on Culinary Herbs 826; 2007. p. 185-92. doi: 10.17660/ActaHortic.2009.826.25.

32. Kumar P, Rana PK, Himshikha, Singhal VK, Gupta RC. Chromosome numbers, characterization of chromosomal pairing during meiosis, origin and natural propagation in polyploid cytotypes (4x,5x and 6x) of Agrimonia eupatoria L. (Rosaceae) in northwest Himalayas (India). Protoplasma. 2014;251(4):781-95. doi: 10.1007/s00709-013-0581-0.

33. Ody P. The Complete Guide to Medicinal Herbal: Herbal Remedies for Common Ailments. London: Dorling Kindersley, 2000.

34. Akbarzadeh T, Sabourian R, Saeedi M, Rezaeizadeh H, Khanavi M, Shams Ardekani MR. Liver tonics: review of plants used in Iranian traditional medicine. Asian Pac J Trop Biomed. 2015;5(3):170-81. doi: 10.1016/s22211691(15)30002-2.

35. Asadi-Samani M, Kafash-Farkhad N, Azimi N, Fasihi A, Alinia-Ahandani E, Rafieian-Kopaei M. Medicinal plants with hepatoprotective activity in Iranian folk medicine. Asian Pac J Trop Biomed. 2015;5(2):146-57. doi: 10.1016/ s2221-1691(15)30159-3.

36. Alam S, Khan N, Nasiruddin M. Evaluation of Curative potential of Qurs-e-Rewand (a Unani Formulation) against CCl4 induced hepatotoxicity in Rats. Int J Basic Med Clin Res. 2015;2(5):107-15.

37. Duke JA. Handbook of Medicinal Herbs. CRC Press; 2002.

38. Bonet MA, Vallès J. Ethnobotany of Montseny biosphere reserve (Catalonia, Iberian Peninsula): plants used in veterinary medicine. J Ethnopharmacol. 2007;110(1):13047. doi: 10.1016/j.jep.2006.09.016.

39. Tsirigotis-Maniecka M, Pawlaczyk-Graja I, Ziewiecki R, Balicki S, Matulová M, Capek P, et al. The polyphenolicpolysaccharide complex of Agrimonia eupatoria L. as an indirect thrombin inhibitor - isolation and chemical characterization. Int J Biol Macromol. 2019;125:124-32. doi: $\quad$ 10.1016/j.ijbiomac.2018.12.017.

40. Granica S, Krupa K, Kłębowska A, Kiss AK. Development and validation of HPLC-DAD-CAD-MS(3) method for qualitative and quantitative standardization of polyphenols in Agrimoniae eupatoriae herba (Ph. Eur). J Pharm Biomed Anal. 2013;86:112-22. doi: 10.1016/j.jpba.2013.08.006.

41. Sytar O, Bruckova K, Hunkova E, Zivcak M, Konate K, Brestic M. The application of multiplex fluorimetric sensor for the analysis of flavonoids content in the medicinal herbs family Asteraceae, Lamiaceae, Rosaceae. Biol Res. 2015;48(1):5. doi: 10.1186/0717-6287-48-5.

42. Muruzović M, Mladenović KG, Stefanović OD, Vasić SM, Čomić LR. Extracts of Agrimonia eupatoria L. as sources of biologically active compounds and evaluation of their antioxidant, antimicrobial, and antibiofilm activities. J Food Drug Anal. 2016;24(3):539-47. doi: 10.1016/j. jfda.2016.02.007.

43. Huzio NM, Grytsyk AR. Research of the amino acid composition of Agrimonia eupatoria. The Pharma Innovation Journal. 2015;4(2):28-9.
44. Feng XL, He YB, Liang YZ, Wang YL, Huang LF, Xie JW. Comparative analysis of the volatile components of Agrimonia eupatoria from leaves and roots by gas chromatography-mass spectrometry and multivariate curve resolution. J Anal Methods Chem. 2013;2013:246986. doi: $\quad 10.1155 / 2013 / 246986$.

45. Kuczmannová A, Gál P, Varinská L, Treml J, Kováč I, Novotný M, et al. Agrimonia eupatoria L. and Cynara cardunculus L. water infusions: phenolic profile and comparison of antioxidant activities. Molecules. 2015;20(11):20538-50. doi: $10.3390 /$ molecules201119715.

46. Santos TN, Costa G, Ferreira JP, Liberal J, Francisco V, Paranhos A, et al. Antioxidant, anti-inflammatory, and analgesic activities of Agrimonia eupatoria L. Infusion. Evid Based Complement Alternat Med. 2017;2017:8309894. doi: $10.1155 / 2017 / 8309894$.

47. Lee KY, Hwang L, Jeong EJ, Kim SH, Kim YC, Sung SH. Effect of neuroprotective flavonoids of Agrimonia eupatoria on glutamate-induced oxidative injury to HT22 hippocampal cells. Biosci Biotechnol Biochem. 2010;74(8):1704-6. doi: 10.1271/bbb. 100200 .

48. Correia H, González-Paramás A, Amaral MT, SantosBuelga C, Batista MT. Polyphenolic profile characterization of Agrimonia eupatoria L. by HPLC with different detection devices. Biomed Chromatogr. 2006;20(1):88-94. doi: 10.1002/bmc.533.

49. Bilia AR, Palme E, Marsili A, Pistelli L, Morelli I. A flavonol glycoside from Agrimonia eupatoria. Phytochemistry. 1993;32(4):1078-9. doi: 10.1016/0031-9422(93)85262-P.

50. Kubínová R, Švajdlenka E, Jankovská D. Anticholinesterase, antioxidant activity and phytochemical investigation into aqueous extracts from five species of Agrimonia genus. Nat Prod Res. 2016;30(10):1174-7. doi: 10.1080/14786419.2015.1043552.

51. Ivanova D, Tasinov O, Vankova D, Kiselova-Kaneva Y. Antioxidative potential of Agrimonia eupatoria L. Medicine. 2011;1(1):20-4.

52. Gião MS, Gomes S, Madureira AR, Faria A, Pestana D, Calhau C, et al. Effect of in vitro digestion upon the antioxidant capacity of aqueous extracts of Agrimonia eupatoria, Rubus idaeus, Salvia sp. and Satureja montana. Food Chem. 2012;131(3):761-7. doi: 10.1016/j. foodchem.2011.09.030.

53. Gião MS, Pestana D, Faria A, Guimarães JT, Pintado ME, Calhau C, et al. Effects of extracts of selected medicinal plants upon hepatic oxidative stress. J Med Food. 2010;13(1):131-6. doi: 10.1089/jmf.2008.0323.

54. Bajer T, Adam M, Galla L, Ventura K. Comparison of various extraction techniques for isolation and determination of isoflavonoids in plants. J Sep Sci. 2007;30(1):122-7. doi: 10.1002/jssc.200600306.

55. Santos T, Ferreira JP, Costa G, Caramona M, Batista T, Vitória I, et al. Evaluation of Agrimonia eupatoria L. as analgesic and anti-inflammatory on in vivo models. 6th European Congress of Pharmacology (EPHAR ); 336P Granada Congress and Exhibitions Centre; 2012.

56. Bensch K, Tiralongo J, Schmidt K, Matthias A, Bone KM, Lehmann $\mathrm{R}$, et al. Investigations into the antiadhesive activity of herbal extracts against Campylobacter jejuni. Phytother Res. 2011;25(8):1125-32. doi: 10.1002/ptr.3384.

57. Ghaima KK. Antibacterial and wound healing activity of some Agrimonia eupatoria extracts. Baghdad Sci J. 2013;10(1):152-60. 
58. Kuczmannová A, Balažová A, Račanská E, Kameníková M, Fialová S, Majerník J, et al. Agrimonia eupatoria L. and Cynara cardunculus L. water infusions: comparison of antidiabetic activities. Molecules. 2016;21(5). doi: 10.3390/ molecules21050564.

59. Watkins F, Pendry B, Sanchez-Medina A, Corcoran O. Antimicrobial assays of three native British plants used in Anglo-Saxon medicine for wound healing formulations in 10th century England. J Ethnopharmacol. 2012;144(2):40815. doi: 10.1016/j.jep.2012.09.031.

60. Komiazyk M, Palczewska M, Sitkiewicz I, Pikula S, Groves P. Neutralization of cholera toxin by Rosaceae family plant extracts. BMC Complement Altern Med. 2019;19(1):140. doi: 10.1186/s12906-019-2540-6.

61. Lee KH, Rhee KH. Anti-nociceptive effect of Agrimonia eupatoria extract on a cisplatininduced neuropathic model. Afr J Tradit Complement Altern Med. 2016;13(5):139-44. doi: 10.21010/ajtcam.v13i5.18.

62. Supuka P, Marcinčák S, Popelka P, Petrovič V, Molnár L, Maskalová I, et al. The effects of adding agrimony and sage extracts to water on blood biochemistry and meat quality of broiler chickens. Acta Vet Brno. 2015;84(2):119-24. doi: 10.2754/avb201584020119.

63. Fejerčáková A, Vašková J, Bača M, Vaško L, Marcinčák S, Hertelyová Z, et al. Effect of dietary microbially produced gamma-linolenic acid and plant extracts on enzymatic and non-enzymatic antioxidants in various broiler chicken organs. J Anim Physiol Anim Nutr (Berl). 2014;98(5):8606. doi: 10.1111/jpn.12146.

64. Petrovic V, Marcincak S, Popelka P, Simkova J, Martonova
M, Buleca J, et al. The effect of supplementation of clove and agrimony or clove and lemon balm on growth performance, antioxidant status and selected indices of lipid profile of broiler chickens. J Anim Physiol Anim Nutr (Berl). 2012;96(6):970-7. doi: 10.1111/j.1439-0396.2011.01207.x.

65. Cho YM, Kwon JE, Lee M, Lea Y, Jeon DY, Kim HJ, et al. Agrimonia eupatoria L. (Agrimony) extract alters liver health in subjects with elevated alanine transaminase levels: a controlled, randomized, and double-blind trial. J Med Food. 2018;21(3):282-8. doi: 10.1089/jmf.2017.4054.

66. Hawrelak JA, Myers SP. Effects of two natural medicine formulations on irritable bowel syndrome symptoms: a pilot study. J Altern Complement Med. 2010;16(10):106571. doi: 10.1089/acm.2009.0090.

67. Ivanova D, Tasinov O, Vankova D, Kiselova-Kaneva Y. Agrimonia eupatoria L. extract modulates glutamatecysteine ligase and glutathione peroxidase expression in 3T3-L1 cells. Bulg J Agric Sci. 2013;19(2):171-4.

68. Hou Q, He WJ, Hao HJ, Han QW, Chen L, Dong L, et al. The four-herb Chinese medicine ANBP enhances wound healing and inhibits scar formation via bidirectional regulation of transformation growth factor pathway. PLoS One. 2014;9(12):e112274. doi: 10.1371/journal. pone. 0112274 .

69. Čurlík J, Kolesár M, Durža O, Hiller E. Dandelion (Taraxacum officinale) and Agrimony (Agrimonia eupatoria) as indicators of geogenic contamination of flysch soils in Eastern Slovakia. Arch Environ Contam Toxicol. 2016;70(3):475-86. doi: 10.1007/s00244-015-0206-z. 